\title{
Childhood Periosteal Osteosarcoma
}

National Cancer Institute

\section{Source}

National Cancer Institute. Childhood Periosteal Osteosarcoma. NCI Thesaurus. Code C123398.

A periosteal osteosarcoma occurring in childhood. 